\title{
Resolving the SELEX-LHCb double-charm baryon conflict: the impact of intrinsic heavy-quark hadroproduction and supersymmetric light-front holographic QCD
}

\author{
S. J. Brodsky ${ }^{1}$, S. Groote ${ }^{2, a}$, S. Koshkarev ${ }^{2}$ \\ ${ }^{1}$ SLAC National Accelerator Laboratory, Stanford University, Stanford, CA 94309, USA \\ ${ }^{2}$ Institute of Physics, University of Tartu, 51010 Tartu, Estonia
}

Received: 1 February 2018 / Accepted: 31 May 2018 / Published online: 11 June 2018

(C) The Author(s) 2018

\begin{abstract}
In this paper we show that the intrinsic heavyquark QCD mechanism for the hadroproduction of heavy hadrons at large $x_{F}$ can resolve the apparent conflict between measurements of double-charm baryons by the SELEX fixed-target experiment and the LHCb experiment at the LHC collider. We show that in fact both experiments are compatible, and that both can be correct. The observed spectroscopy of double-charm hadrons is in agreement with the predictions of supersymmetric light front holographic QCD.
\end{abstract}

\section{Introduction}

The first experimental evidence for the existence of doublecharm baryons was published by the SELEX collaboration 15 years ago [1-6]. By utilizing the Fermilab negative and positive charged beams at $600 \mathrm{GeV} / c$ to produce charmed particles in a thin foil of copper or on a diamond target, the SELEX collaboration observed two different decay channels for the $|d c c\rangle$ state at a mass close to $3520 \mathrm{MeV} / c^{2}$.

The SELEX fixed-target experiment measured hadron production in the forward kinematic domain $x_{F}>0.1$. The negative beam composition was about $50 \% \Sigma^{-}$and $50 \%$ $\pi^{-}$, whereas the positive beams were composed of $50 \%$ protons. The experimental data recorded used both positive and negative beams: $67 \%$ of the events were induced by $\Sigma^{-}, 13 \%$ by $\pi^{-}$, and $18 \%$ by protons. In the first observation using the sample of $\Lambda_{c}^{+} \rightarrow p K^{-} \pi^{+}$[7,8] SELEX found a signal of 15.9 events over $6.1 \pm 0.1$ background events in the channel $\Xi_{c c}^{+} \rightarrow \Lambda_{c}^{+} K^{-} \pi^{+}$[1]. To complement this result, SELEX published an observation of 5.62 signal events over $1.38 \pm 0.13$ background events for the decay mode $\Xi_{c c}^{+} \rightarrow p D K^{-}$from a sample of $D^{+} \rightarrow K^{-} \pi^{+} \pi^{+}$ decays [4].

a e-mail: groote@ut.ee
Two charm quarks will not be produced at high $x_{F}$ from DGLAP evolution [9-11] or perturbative gluon splitting $g \rightarrow g+g \rightarrow(\bar{c} c)+(\bar{c} c)$ [11-13]. Therefore, the observation by SELEX of a double-charm baryon $|q c c\rangle$ at a large mean value for $x_{F}$ and a relatively small mean transverse momentum can raise skepticism. However, the $\Lambda_{c}(u d c)$ and the $\Lambda_{b}(u d b)$ were both discovered at the ISR at high $x_{F}$ [14-16]. In addition, the NA3 experiment measured both single-quarkonium hadroproduction $\pi A \rightarrow J / \psi X$ [17] and double-quarkonium hadroproduction $\pi A \rightarrow J / \psi J / \psi X[18]$ at high $x_{F}$. In fact, all of the $\pi A \rightarrow J / \psi J / \psi X$ events were observed by NA3 with total $x_{F}>0.4$.

The existence of heavy quarks at large light-front (LF) momentum fraction $x$ in the proton's light-front wavefunction is in fact predicted by QCD if one analyzes the higher Fock states $|u u d c \bar{c}\rangle$ and $|u u d c \bar{c} c \bar{c}\rangle$ in the hadronic eigenstate; i.e., Fock states where the heavy quark pairs are multiconnected to the valence quarks. LF wavefunctions, the eigensolutions of the QCD LF Hamiltonian, are defined at fixed LF time $\tau=t+z / c$, and are thus off-shell in invariant mass. For example, in QED, positronium has an analogous $\left|e^{+} e^{-} \mu^{+} \mu^{-}\right\rangle$Fock state due to the insertion of light-bylight scattering in the positronium self-energy amplitude. In such an "intrinsic charm" Fock state $|u u d c \bar{c}\rangle$, the maximum kinematic configuration occurs at minimum invariant mass, where all quarks are at rest in the hadron's rest frame; i.e., at equal rapidity in the moving hadron. Equal rapidity implies $x_{i} \propto\left(m^{2}+\mathbf{k}_{\perp}{ }^{2}\right)^{1 / 2}$ for each quark, so that the heavy quarks in the Fock state carry most of the hadron's LF momentum. The operator product expansion predicts that the probability of intrinsic heavy-quark Fock states $|u u d Q \bar{Q}\rangle$ to scale as $1 / m_{Q}^{2}$ due to the non-Abelian couplings of QCD $[19,20]$.

If such a Fock state interacts in a hadronic collision, the comoving $u d c$ of the projectile proton can readily coalesce 
into the $\Lambda_{c}(u d c)$ bound state with $x_{F}^{\Lambda}=x_{c}+x_{u}+x_{d}>0.4$. Similarly, it is natural to hadro-produce a double-charm baryon $|q c c\rangle$ at high $x_{F}$ from the materialization of the double-intrinsic charm $|u u d c \bar{c} c \bar{c}\rangle$ Fock state of the projectile proton, since the $q c c$ quarks can coalesce at the same rapidity [21]. The production rate for double-charm baryons is thus related to double quarkonium hadroproduction as observed by NA3, since they both originate from the same doubleintrinsic charm Fock state of the projectile.

Recently, the LHCb collaboration published an observation of $313 \pm 33$ events of $\Xi_{c c}^{++} \rightarrow \Lambda_{c}^{+} K^{-} \pi^{+} \pi^{+}$in a $13 \mathrm{TeV}$ sample at the LHC and $113 \pm 21$ events in a $8 \mathrm{TeV}$ sample at mass $3621.40 \pm 0.72$ (stat) \pm 0.27 (sys) $\pm 0.14\left(\Lambda_{c}^{+}\right) \mathrm{MeV} / c^{2}$, corresponding to 1.7 and $2 \mathrm{fb}^{-1}$, respectively [22]. LHCb reported that the mass difference between the $\Xi_{c c}^{+}(d c c)$ candidate reported by SELEX and the $\Xi_{c c}^{++}(u c c)$ state reported by $\mathrm{LHCb}$ was $103 \mathrm{MeV} / c^{2}$, so these states cannot be readily interpreted as an isospin doublet since one would expect a mass difference of isospin partners to differ by only a few $\mathrm{MeV} / c^{2}$. Note, though, that the upper limit of the $x_{F}$ range at the LHCb collider experiment is given by $x_{F} \approx 0.15$ and $x_{F} \approx 0.09$ for the 8 and $13 \mathrm{GeV}$ analysis, respectively (see discussion in Sec. 5). In contrast to this, the $x_{F}$ range at the SELEX fixed-target experiment starts at $x_{F}=0.1$, nearly complementary to the acceptance for the LHCb. In this paper we review the hadroproduction mechanisms of double-charm baryons for the different experimental environments and reinterpret the SELEX and LHCb results.

\section{Production rate and the kinematics of the $\Xi_{c c}^{+}$for the SELEX experiment}

The SELEX collaboration did not provide the absolute production rate for the double charmed baryon state $|d c c\rangle$. Fortunately, this rate can be compared to that of $\Lambda_{c}^{+}$baryon. The production ratio $R_{\Lambda_{c}^{+}}$measured by SELEX is given by

$R_{\Lambda_{c}^{+}}^{\mathrm{SELEX}}=\frac{\sigma\left(\Xi_{c c}^{+}\right) \cdot \operatorname{Br}\left(\Xi_{c c}^{+} \rightarrow \Lambda_{c}^{+} K^{-} \pi^{+}\right)}{\sigma\left(\Lambda_{c}^{+}\right)}=\frac{N_{\Xi_{c c}^{+}}}{\epsilon_{+}} \cdot \frac{\epsilon_{\Lambda_{c}^{+}}}{N_{\Lambda_{c}^{+}}}$,

where $N$ is the number of events in the respective sample, and the reconstruction efficiency of $\Xi_{c c}^{+}$is given by $\epsilon_{+} \approx 11 \%$ [1]. The central value for the number $N_{\Lambda_{c}^{+}} / \epsilon_{\Lambda_{c}^{+}}$ of reconstructed $\Lambda_{c}^{+}$baryon events reported in Ref. [23] lies between 13326 and 10010 according to whether the lowest bin with $x_{F} \in[0.125,0.175]$ is taken into account or not. Therefore, we obtain

$R_{\Lambda_{c}^{+}}^{\mathrm{SELEX}} \approx 0.012-0.014$.

If we take into account the intrinsic charm mechanism, the reconstruction efficiency of $\Xi_{c c}^{+}$will grow at least by a factor of 2.3 mainly because the $x_{F}$ distribution predicted by intrinsic charm at large Feynman $x_{F}$ is well matched to the acceptance of the SELEX fixed-target experiment [2] (cf. Ref. [24]). As a consequence, $R_{\Lambda_{c}^{+}}^{\mathrm{SELEX}}$ can be even smaller, $R_{\Lambda_{c}^{+}}^{\text {SELE }} \sim(0.5-0.6) \times 10^{-3}$.

By using the pion beam at 150 and $280 \mathrm{GeV} / c$ at CERN incident on hydrogen and platinum targets, the NA3 experiment provided data on double $J / \psi$ production with very similar features: a value for the ratio $\sigma(\psi \psi) / \sigma(\psi)=$ $(3 \pm 1) \times 10^{-4}$ one or two orders of magnitude higher than the conventional prediction of perturbative QCD [25], values $x_{\psi \psi}>0.6$ at $150 \mathrm{GeV} / c$ and $x_{\psi \psi}>0.4$ at $280 \mathrm{GeV} / c$ for the total Feynman- $x_{F}$ and total transverse momentum $p_{T, \psi \psi}=0.9 \pm 0.1 \mathrm{GeV} / c$ of the $J / \psi$ pair $[18,26]$.

Note that gluon-gluon fusion, quark-antiquark annihilation and the gluon splitting mechanism of perturbative QCD can explain neither the NA3 cross section nor the $x_{F}$ distribution (see Ref. [27] and references therein). Both double $J / \psi$ and doubly-charmed baryons could be produced perturbatively by mechanisms such as double parton scattering or some kind of ladder diagram where e.g. gluons emitted from the projectile and the target both split into $Q \bar{Q}$ pairs, one heavy quark from each pair being connected by an intermediate gluon. This is of course not the same as double parton scattering but leads to the same outcome in terms of producing two $Q \bar{Q}$ pairs in the same hard scattering. However, these pairs would typically have lower average energies, softer $p_{T}$ distributions and narrower $x_{F}$ ranges, particularly at fixedtarget energies. They are also reduced in yield by additional factors of the strong coupling constant. However, intrinsic charm has none of these deficiencies but just a lower probability for the production of the second $Q \bar{Q}$ pair, while it still produces relatively large $x_{F}$ heavy flavor quarks.

It is clearly of interest to relate the production of the $\Xi_{c c}^{+}$at the SELEX experiment with the production of double $J / \psi$ production at the NA3 experiment. Unfortunately, it is not possible to compare the two results directly. However, we are able to compare the following ratios $R=\sigma(c \bar{c} c \bar{c}) / \sigma(c \bar{c})$ :

$R^{\mathrm{SELEX}}=R_{\Lambda_{c}^{+}} \times \frac{f\left(c \rightarrow \Lambda_{c}^{+}\right)}{f_{\Xi_{c c}}} \sim(1-4) \times 10^{-3}$

and

$R^{\mathrm{NA} 3}=\frac{\sigma(\psi \psi)}{\sigma(\psi)} \times \frac{f_{\psi}}{f_{\psi / \pi}^{2}} \sim 2 \times 10^{-2}$,

where $f_{\psi / \pi} \approx 0.03$ is the fragmentation rate of the intrinsic charm state of the pion into $J / \psi[28]$ and $f_{\psi} \approx 0.06$ is the perturbative QCD fragmentation rate into $J / \psi$ [29]. $f_{\Xi_{c c}} \approx 0.25[30]$ represents the fraction of double $c \bar{c}$ pairs producing the sum of single-charged baryons $\Xi_{c c}^{+}$and double-charged baryons $\Xi_{c c}^{++}$, but this fraction cannot be less than the fraction to produce $J / \psi$. Therefore, $R^{\text {SELEX }}$ should not be larger than $10^{-2}$. The SELEX production ratio is thus 
in approximate consistency with the complementary measurement of the double $J / \psi$ production by the NA3 experiment. It is interesting to note that the intrinsic charm mechanism predicts $\left\langle x_{F}\left(\Xi_{c c}\right)\right\rangle=0.33$, as shown in Ref. [30]. This is in excellent agreement with the value $\left\langle x_{F}\left(\Xi_{c c}^{+}\right)\right\rangle \sim 0.33$ measured by the SELEX experiment.

It should be emphasized that SELEX observed the weak decay of the $3520 \mathrm{MeV}$ double-charm baryon in two different decay channels, namely $\Xi_{c c}^{+}(3519 \pm 1) \rightarrow \Lambda_{c}^{+} K^{-} \pi^{+}$and $\Xi_{c c}^{+}(3518 \pm 3) \rightarrow p D^{+} \pi^{-}$with statistical significances of $6.3 \sigma$ and $4.8 \sigma$, respectively. The probability that these two signals are statistical fluctuations is extremely small.

\section{Mass difference}

In order to resolve the discrepancy between the results from SELEX and LHCb we will utilize the predictions of the supersymmetric light front holographic QCD (SUSY LFHQCD). This approach was developed by imposing the constraints from the superconformal algebraic structure on LFHQCD for massless quarks [31]. As has been shown in Refs. [31,32], supersymmetry holds to a good approximation, even if conformal symmetry is strongly broken by the heavy quark mass.

Note that the $3_{C}+\overline{3}_{C}$ diquark structure of the $\Xi_{c c}^{+}$can be written explicitly as $|[d c] c\rangle$ state $[33,34]$. The production of the double-charm baryon $\Xi_{[d c] c}^{+}$with $[d c]$ in a spin-singlet state is natural in the SELEX fixed target experiment since it has acceptance at high $x_{F}$, i.e., in the realm of intrinsic charm; the $[d c] c$ configuration can easily re-coalesce from a higher Fock state of the proton such as $|u u d c \bar{c} c \bar{c}\rangle$. In contrast, the production of this state is likely to be suppressed in $q \bar{q} \rightarrow c \bar{c} c \bar{c}$ or $g g \rightarrow c \bar{c} c \bar{c}$ reactions at the LHCb. Thus $\mathrm{LHCb}$ has most likely observed the double-charm baryon state $|u(c c)\rangle$, as will be explained in the next section. ${ }^{1}$ The mass difference between the $|[d c] c\rangle$ and the $|u(c c)\rangle$ states is due to the hyperfine interaction between the quarks.

Supersymmetric light front holographic QCD, if extended to the case of two heavy quarks, predicts that the mass of the spin-1/2 baryon should be the same as the mass of $h_{c}(1 P)(3525)$ meson [32]. This is well compatible with the SELEX measurement of $3520.2 \pm 0.7 \mathrm{MeV} / c^{2}$ for the $\Xi_{c c}^{+}(d[c c])$, although the uncertainty of SUSY LFHQCD predictions is at least of the order of $100 \mathrm{MeV}$. Indeed, the mass of the $|u(c c)\rangle$ state is predicted to be the same as that of the $\chi_{c 2}(1 P)(3556)$ meson, which is in turn lower than the $\mathrm{LHCb}$ result of $3621.40 \pm 0.72$ (stat) \pm 0.27 (sys) \pm $0.14\left(\Lambda_{c}^{+}\right) \mathrm{MeV} / c^{2}$ for the $\Xi_{c c}^{++}$.

Supersymmetric LFHQCD is based on and best tested in the chiral limit of QCD, where all quarks are massless.

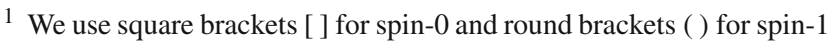
internal states.
}

The mass difference between the $h_{c}(1 P)$ and the $\chi_{c 2}(1 P)$ is mainly due to the hyperfine splitting between the two charm quarks, and hence very small. In the baryon there is also the larger spin-spin interaction between the $c$ and a light quark. By comparing hadron masses with light and charmed quarks, one can estimate the strength of this additional, the supersymmetry-breaking interaction in the range 84-136 MeV $/ c^{2}[32,35]$, which is well compatible with the mass difference between the SELEX and the LHCb states.

\section{The SELEX state at the LHCb}

In the previous section we identified the SELEX state as a $|[d c] c\rangle$ state and the LHCb state as a $|u(c c)\rangle$ state of the double charmed baryon. While the SELEX state is definitely a spin- $1 / 2$ state, both $J^{P}=1 / 2^{+}$and $J^{P}=3 / 2^{+}$are possible assignments for the $\mathrm{LHCb}$ state. As becomes clear in the following, $J^{P}=1 / 2$ is favored by the LHCb mass measurement and the very suppressed radiative decay to $|[q c] c\rangle+\gamma$. Based on the Heavy Quark Effective Theory (HQET), in Ref. [36] the baryon masses are estimated to be $3610 \mathrm{MeV} / c^{2}$ for the spin- $1 / 2$ state $|u(c c)\rangle_{1 / 2^{+}}$and $3680 \mathrm{MeV} / c^{2}$ for the spin-3/2 state $|u(c c)\rangle_{3 / 2^{+}}$. For a qualitative estimate one can also compare with the nucleon states where the lowest mass $I=1 / 2, J^{P}=3 / 2^{+}$state is the $N(1720)$ which is considerably more massive than the proton.

It is interesting to analyze the ability of the $\mathrm{LHCb}$ experiment to observe the $|d c c\rangle$ state, i.e. $\Xi_{c c}^{+}$. Note that in a sample corresponding to $0.65 \mathrm{fb}^{-1}$ of integrated luminosity at $7 \mathrm{TeV}$, in case of the decay process $\Xi_{c c}^{+} \rightarrow \Lambda_{c}^{+} K^{-} \pi^{+}$the $\mathrm{LHCb}$ collaboration published an upper limit for the ratio $\sigma\left(\Xi_{c c}^{+}\right)$. $\operatorname{Br}\left(\Xi_{c c}^{+} \rightarrow \Lambda_{c}^{+} K^{-} \pi^{+}\right) / \sigma\left(\Lambda_{c}^{+}\right)$of $1.5 \times 10^{-2}$ and $3.9 \times 10^{-4}$ for the lifetimes $100 \mathrm{fs}$ and $400 \mathrm{fs}$, respectively [37]. In case of the decay $\Xi_{c c}^{++} \rightarrow \Lambda_{c}^{+} K^{-} \pi^{+} \pi^{+}$analyzed in Ref. [22], one expects a larger lifetime, $\tau\left(\Xi_{c c}^{++}\right) / \tau\left(\Xi_{c c}^{+}\right) \approx 2.5-4$ (cf. Refs. [25,38]). Taking into account that the cuts were optimized for the lifetime of $333 \mathrm{fs}$ and that the minimum lifetime reached by the $\mathrm{LHCb}$ is about three times larger than the lifetime $\tau\left(\Xi_{c c}^{+}\right)<33$ fs measured by the SELEX at $90 \%$ confidence level, the $\mathrm{LHCb}$ provided an analysis which was outside the signal region for $\Xi_{c c}^{+}$.

It is also of interest to analyze the expectation of the production ratio between states. As we discussed above, the production of the double-charm baryons at the $\mathrm{LHCb}$ is due to $q \bar{q} \rightarrow c \bar{c} c \bar{c}$ or $g g \rightarrow c \bar{c} c \bar{c}$ reactions with the following fragmentation of the $c c$-diquark into the double-charm baryons. Due to the Pauli principle the $c c$-diquark has to be a state with spin 1 or higher, leading to a state $|q(c c)\rangle$ with higher mass. The normalization of the fragmentation of the $c c$-diquark into the double-charm baryons is unknown. However, we are still able to provide some quantitative analysis. The fragmentation function is proportional to the wave function at the origin. 
The color-anti-triplet wave function can be estimated on the basis of information about the color-singlet wave function, $\left|R(0)[c c]_{\overline{\mathbf{3}}}\right| \sim\left|R(0)[c \bar{c}]_{\mathbf{1}}\right|$. It is clear that the $|[q c] c\rangle$ states cannot be produced through the fragmentation mechanism on the LHCb.

\section{Suppression of the radiative decay}

An important issue is the rate for the heavier $|u(c c)\rangle$ state to decay radiatively to the spin-1/2 ground state $|[u c] c\rangle_{1 / 2^{+}}$. However, LHCb explicitly observed that the state they discovered decays weakly which means that the radiative decay of the LHCb state at $3621 \mathrm{MeV}$ has to be strongly suppressed: The radiative lifetime has to be longer than approximately $50 \mathrm{fs}$ in order that at least some of the $\mathrm{LHCb} 3621 \mathrm{MeV}$ states would have survived and observed to decay weakly. A lifetime of $50 \mathrm{fs}$ means that the transition rate $\Gamma(3621 \rightarrow$ $3520+\gamma)$ has to be less than $0.01 \mathrm{eV}$. The energy of the photon emitted by the radiative transition is $\omega=101 \mathrm{MeV}$. The dependence of the transition rate on $\omega$ comes from (a) the phase space of the final state and (b) the dynamical suppression from the square of the matrix element of the electromagnetic current connecting the initial and final eigenstates.

For comparison, one can consider the measured radiative decay rate for $J / \psi \rightarrow \eta_{c} \gamma$ with photon energy $\omega=115 \mathrm{MeV}$ [39]. The measured radiative decay rate for $J / \psi \rightarrow \eta_{c} \gamma$ is $\Gamma^{\exp }=1.13 \pm 0.35 \mathrm{keV}$. Note that the spatial wavefunctions of the $J / \psi$ and the $\eta_{c}$ are almost identical. In contrast, the initial and final state wavefunctions are very different. In the case of transition between the doublecharm baryons $|q(c c)\rangle_{3 / 2^{+}} \rightarrow|[q c] c\rangle_{1 / 2^{+}}+\gamma$ the amplitude for radiative decay thus involves the matrix element $\left\langle f\left|\mathbf{J}_{\mathrm{em}}\right| i\right\rangle$ of the electromagnetic current between highly orthogonal hadronic eigenstates. In particular, the emission of the $\omega=101 \mathrm{MeV}$ photon also has to interchange one of the charm quarks in the spin-1 $(c c)$ diquark with the light quark $q$ to form the spin-0 $[q c]$ diquark.

The matrix element in the LF framework involves the overlap of the current with nearly orthogonal three-body light-front wavefunctions $\psi_{i}\left(x, \mathbf{k}_{\perp}\right)$ and $\psi_{f}\left(x, \mathbf{k}_{\perp}\right)$ at the small $101 \mathrm{MeV}$ momentum transfer. The matrix element thus must vanish as $\left(\omega r_{c c}\right)^{3}$, where $r_{c c}$ characterizes the size of the radial wavefunctions. This gives a strong suppression of the rate of $\left(\omega r_{c c}\right)^{6}$ relative to that of radiative charmonium decays.

According to Ref. [40], $r_{J / \psi} \sim 0.39 \mathrm{fm} \simeq 2 \mathrm{GeV}^{-1}$. As a first estimate, we will assume that the characteristic radial size of the charm diquark $c c$ in the double-charm baryons is the same as that of the $J / \psi, r_{c c} \sim r_{J / \psi}$, resulting in $\left(\omega r_{c c}\right)^{6} \sim 6 \times 10^{-5}$. In comparison with the $1 \mathrm{keV}$ $J / \psi \rightarrow \eta_{c} \gamma$ decay width, this would give a radiative transition rate for $|q(c c)\rangle_{3 / 2^{+}} \rightarrow|[q c] c\rangle_{1 / 2^{+}}+\gamma$ of order
$0.06 \mathrm{eV}$ and thus a radiative transition lifetime of $10 \mathrm{fs}$. A radiative lifetime of this order would not prevent the $\mathrm{LHCb}$ from observing the weak decay of the $|q(c c)\rangle$ double-charm baryon at $3621 \mathrm{MeV}$. In this case LHCb might be able to observe some radiative events $|[q c] c\rangle_{1 / 2^{+}}+\gamma$, where the $|[q c] c\rangle_{1 / 2^{+}}$at $3520 \mathrm{MeV}$ decays weakly.

It is also possible that the $3621 \mathrm{MeV}$ state observed by $\mathrm{LHCb}$ is a $J^{P}=1 / 2^{+}$double-charm baryon state $|q(c c)\rangle_{1 / 2^{+}}$, rather than $J^{P}=3 / 2^{+}$since we have assumed that it is a bound state of a spin-1/2 quark and a spin-1 (cc) diquark. In this case the spin-1 photon needs to be emitted with orbital angular momentum $L=1$ to conserve parity in the radiative decay of the $3621 \mathrm{MeV}$ state $|q(c c)\rangle_{1 / 2^{+}}$to the $3520 \mathrm{MeV}$ state $|[q c] c\rangle_{1 / 2^{+}}$. This would give an additional factor of $v^{2}$ in the phase space for the radiative transition rate where $v \simeq \omega / M \sim 1 / 35$ is the recoil velocity of the $3520 \mathrm{MeV}$ double-charm baryon. This additional suppression of the rate implies that the lifetime of the radiative transition would then be increased to be of order $12000 \mathrm{fs}$.

We have also done a comparison with the radiative transition rates between the $J^{P}=1^{-}$charmonium $S$ states analyzed in the classic paper by Feinberg and Sucher [41], such as the estimated $10 \mathrm{keV}$ transition rate between the $\psi(2 S)(3686 \mathrm{MeV})$ and the $J / \psi(3097 \mathrm{MeV})$. The transition energy in our case is $101 \mathrm{MeV}$, compared to the transition energy between the $\psi(2 S)(3686 \mathrm{MeV})$ and the $J / \psi(3097 \mathrm{MeV})$ of $589 \mathrm{MeV}$ - a relative reduction in the photon transition energy of $101 / 589 \sim 1 / 6$. This factor enters at the third power in the matrix element of the spin current in Eq. (9) of Ref. [41] through the overlap of radial wavefunctions, i.e. the spherical Bessel function $j_{0}(k r / 2)$. In addition, one has to take into account the additional suppression of electromagnetic transitions between the $q(c c)$ and $[q c] c$ configurations when extrapolating to double-charm baryons.

The above discussion is clearly only a first estimate. A rigorous treatment of the transition radiative decay rate between double-charm baryons with diquarks of different spin and composition is clearly necessary.

We also note that $\mathrm{LHCb}$ may be be able to detect radiative transitions involving double-charm baryons which have higher masses and higher spin. The observation of the $3520 \mathrm{MeV}$ state $|[u c] c\rangle_{1 / 2^{+}}$with a significant transverse momentum kick from photon emission from a heavy double charm state would be a important confirmation of our picture. Because of this, we suggest that $\mathrm{LHCb}$ conduct such a search.

Still, there might be intrinsic charm in the wave function at LHC. To analyze this opportunity we will follow a similar discussion given in Ref. [30]. The contribution from the double intrinsic charm should be suppressed due to the kinematics of the $\mathrm{LHCb}$ experiment: Making the naive assumption that the momentum is split evenly between all final states and taking into account that the hadron identification efficiency 
for pions and kaons is degraded above $100 \mathrm{GeV} / c[42,43]$, the analysis loses sensitivity around $p\left(\Xi_{c c}^{++}\right) \approx 600 \mathrm{GeV} / c$, i.e. $x_{F} \approx 0.15$ and $x_{F} \approx 0.09$ for the 8 and $13 \mathrm{GeV}$ analysis, respectively. This range of values for $x_{F}$ corresponds to the rapidity region $2<y<5$ in which the LHCb detector operates [44]. Note that in contrast to SELEX, LHCb is a collider experiment where the acceptance excludes the detection of events close to the beam axis.

\section{Summary}

Using both theoretical and experimental arguments, we have shown that the SELEX and the LHCb results for the production of doubly charmed baryons can both be correct. We have compared the data for double $J / \psi$ production observed by the NA3 experiment and the SELEX result for $\Xi_{c c}^{+}$production at high Feynman- $x_{F}$. We have found that the NA3 data strongly complement the SELEX production rate for the spin-1/2 $|[d c] c\rangle$ state. In contrast, LHCb has most likely discovered the the heavier $|u(c c)\rangle$ produced by gluon-gluon fusion $g g \rightarrow c \bar{c} c \bar{c}$ at $x_{F} \sim 0$. The application of supersymmetric algebra to hadron spectroscopy, together with the intrinsic heavy-quark QCD mechanism for the hadroproduction of heavy hadrons at large $x_{F}$, can thus resolve the apparent conflict between measurements of double-charm baryons by the SELEX fixed-target experiment and the LHCb experiment at the LHC collider. The mass difference of the two double-charm baryons reflects the distinct spins of the underlying diquarks.

An important conclusion from our study is that the natural kinematic domain for producing novel hadronic bound states, such as multi-heavy quark hadrons and the tetraquarks predicted by superconformal algebra, is large Feynman $x_{F}$. In this domain, the constituents of the higher Fock states of the projectile, which are comoving at the same rapidity, can coalesce to produce a wide variety of color-singlet hadrons.

Our paper shows why the state $|[q c] c\rangle_{1 / 2^{+}}$is favorably produced at high $x_{F}$, within the kinematics of the SELEX acceptance, and conversely, why its production is unfavorable in the LHC acceptance. There are also very strong upper limits for the production of double-charm baryons in forward photoproduction from duality which explains why the FOCUS experiment does not observe any signal for double charm baryons [45].

These observations indicate the importance of the high $x_{F}$ domain and fixed-target LHC experiments such as SMOG@LHCb [46,47] and AFTER@LHC [48,49] for observing the hadroproduction of exotic heavy quark states. As explained above, one also can understand why the radiative transition $3621|(c c) q\rangle \rightarrow 3520|[q c] c\rangle+\gamma$ is strongly suppressed due to the very small overlap of their respective radial wavefunctions.
The SELEX experiment measured the lifetime $\tau\left(\Xi_{c c}^{+}\right)<$ $33 \mathrm{fs}$ at $90 \%$ confidence level. Using the ratio $\tau\left(\Xi_{c c}^{++}\right) / \tau\left(\Xi_{c c}^{+}\right)$ $\approx 2.5-4$ we can assume $\tau\left(\Xi_{c c}^{++}\right) \approx 100 \mathrm{fs}$. This value stands in contrast to the minimum values theoretically predicted as $\tau\left(\Xi_{c c}^{+}\right) \approx 53 \mathrm{fs}$ and $\tau\left(\Xi_{c c}^{++}\right) \approx 185 \mathrm{fs}$ [38]. Therefore, definitive measurements of the lifetime will provide another test for the SELEX data.

Acknowledgements This research was supported by the Estonian Research Council under Grant No. IUT2-27 and by the Department of Energy under Contract No. DE-AC02-76SF00515. The authors would like to thank H.G. Dosch and G.F. de Téramond for critical comments.

Open Access This article is distributed under the terms of the Creative Commons Attribution 4.0 International License (http://creativecomm ons.org/licenses/by/4.0/), which permits unrestricted use, distribution, and reproduction in any medium, provided you give appropriate credit to the original author(s) and the source, provide a link to the Creative Commons license, and indicate if changes were made.

Funded by SCOAP ${ }^{3}$.

\section{References}

1. M. Mattson et al., [SELEX Collaboration]. Phys. Rev. Lett. 89, 112001 (2002)

2. M. Mattson, Ph.D. thesis, Carnegie Mellon University (2002)

3. M.A. Moinester et al. [SELEX Collaboration], Czech. J. Phys. 53, B201 (2003)

4. A. Ocherashvili et al. [SELEX Collaboration], Phys. Lett. B 628, 18 (2005)

5. J. Engelfried [SELEX Collaboration], Nucl. Phys. A 752, 121 (2005)

6. J. Engelfried [SELEX Collaboration], Proceedings of the International Conference on Heavy Quarks and Leptons (HQL 06), Munich, Germany, 16-20 October 2006, eConf C 0610161, 003 (2006)

7. A. Kushnirenko et al. [SELEX Collaboration], Phys. Rev. Lett. 86, $5243(2001)$

8. A. Kushnirenko, Ph.D. thesis, Carnegie Mellon University (2000)

9. V.N. Gribov, L.N. Lipatov, Sov. J. Nucl. Phys. 15, 438 (1972) [Yad. Fiz. 15, 781 (1972)]

10. Y.L. Dokshitzer, Sov. Phys. JETP 46, 641 (1977) [Zh. Eksp. Teor. Fiz. 73, 1216 (1977)]

11. G. Altarelli, G. Parisi, Nucl. Phys. B 126, 298 (1977)

12. G. Curci, W. Furmanski, R. Petronzio, Nucl. Phys. B 175, 27 (1980)

13. W. Furmanski, R. Petronzio, Phys. Lett. 97B, 437 (1980)

14. W.S. Lockman, T. Meyer, J. Rander, P. Schlein, R. Webb, S. Erhan, J. Zsembery, Phys. Lett. B 85, 443 (1979)

15. P. Chauvat et al. [R608 Collaboration], Phys. Lett. B 199, 304 (1987)

16. G. Bari et al., Nuovo Cim. A 104, 1787 (1991)

17. J. Badier et al. [NA3 Collaboration], Z. Phys. C 20, 101 (1983)

18. J. Badier et al. [NA3 Collaboration], Phys. Lett. B 114, 457 (1982)

19. S.J. Brodsky, J.C. Collins, S.D. Ellis, J.F. Gunion, A.H. Mueller, Snowmass Summer Study, 0227 (1984)

20. M. Franz, M.V. Polyakov, K. Goeke, Phys. Rev. D 62, 074024 (2000)

21. S. Brodsky, S. Gardner, Contribution to the workshop "Physics at the Intensity Frontier", Nov. 30th-Dec. 2nd, 2011. (SLAC-PUB, Washington, D.C., 2012), pp. 14828

22. R. Aaij et al. [LHCb Collaboration], Phys. Rev. Lett. 119, 112001 (2017) 
23. F.G. Garcia et al. [SELEX Collaboration], Phys. Lett. B 528, 49 (2002)

24. S. Groote, S. Koshkarev, Eur. Phys. J. C 77, 509 (2017)

25. V.V. Kiselev, A.K. Likhoded, Phys. Usp. 45, 455 (2002) [Usp. Fiz. Nauk 172, 497 (2002)]

26. J. Badier et al. [NA3 Collaboration], Phys. Lett. B 158, 85 (1985)

27. S. Koshkarev, S. Groote, J. Phys. Conf. Ser. 938(1), 012054 (2017)

28. R. Vogt, S.J. Brodsky, Phys. Lett. B 349, 569 (1995)

29. M. Mangano, H. Satz, U. Wiedermann (eds.) CERN Yellow report, CERN-2004-009 (2004)

30. S. Koshkarev, V. Anikeev, Phys. Lett. B 765, 171 (2017)

31. H.G. Dosch, G.F. de Téramond, S.J. Brodsky, Phys. Rev. D 91, 085016 (2015)

32. H.G. Dosch, G.F. de Téramond, S.J. Brodsky, Phys. Rev. D 92, 074010 (2015)

33. S. Koshkarev, S. Groote. arXiv:1803.07034 [hep-ph]

34. H.G. Dosch, G.F. de Téramond, S.J. Brodsky, Phys. Rev. D 95, 034016 (2017)

35. S.J. Brodsky, G.F. de Téramond, H.G. Dosch, C. Lorcé, Phys. Lett. B 759, 171 (2016)

36. J.G. Körner, M. Krämer, D. Pirjol, Prog. Part. Nucl. Phys. 33, 787 (1994)

37. R. Aaij et al. [LHCb Collaboration], JHEP 1312, 090 (2013)
38. M. Karliner, J.L. Rosner, Phys. Rev. D 90, 094007 (2014)

39. D. Ebert, R.N. Faustov, V.O. Galkin, Phys. Rev. D 67, 014027 (2003)

40. K. Nochi, T. Kawanai, S. Sasaki, Phys. Rev. D 94(11), 114514 (2016)

41. G. Feinberg, J. Sucher, Phys. Rev. Lett. 35, 1740 (1975)

42. M. Adinolfi et al. [LHCb RICH Group], Eur. Phys. J. C 73, 2431 (2013)

43. A. Papanestis et al. [LHCb RICH Collaboration], Nucl. Instrum. Meth. A 876, 221 (2017)

44. A.A. Alves Jr. et al. [LHCb Collaboration], JINST 3, S08005 (2008)

45. S.P. Ratti, Nucl. Phys. Proc. Suppl. 115, 33 (2003)

46. R. Aaij et al. [LHCb Collaboration], JINST 7, P01010 (2012)

47. R. Aaij et al. [LHCb Collaboration], Int. J. Mod. Phys. A 30(07), 1530022 (2015)

48. S.J. Brodsky, F. Fleuret, C. Hadjidakis, J.P. Lansberg, Phys. Rept. 522, 239 (2013)

49. S. Koshkarev, Acta Phys. Polon. B 48, 163 (2017) 